\title{
Operationalizing Neuroimaging for Disorders of Consciousness: The Canadian Context
}

\author{
Judy Illes, Adrian M. Owen, Adrian C. Byram, and the MCS Neuroimaging Workgroup
}

Keywords: Brain injury, functional neuroimaging, quality of life

doi:10.1017/cjn.2015.395

Can J Neurol Sci. 2016; 43: 578-580

Severe brain injury affects tens of thousands of Canadians each year, leaving a small but significant proportion who survive the acute phase in a vegetative state (VS) or minimally conscious state (MCS). Patients in both VS and MCS have diurnal patterns of sleep-wake cycles, but have no behavioral response (VS) or only an inconsistent response (MCS) to stimuli. New technological capabilities offer a window into these disorders-not only changing our understanding of the levels of consciousness, but also enabling the potential for better diagnosis. For example, studies using functional magnetic resonance imaging, now extending over a decade and involving many participants, have revealed that a small minority of patients with severe disorders of consciousness (DoC) can modulate their neural states and thereby demonstrate a limited degree of awareness and even reasoning comparable to unaffected persons. ${ }^{1,2}$

The governments of Canada, its provinces, and territories have recognized and enshrined in legislation and the Constitution moral obligations to persons with disabilities based on the fundamental human rights of autonomy and equality, among others. The right to equality and nondiscrimination is reflected in the Canadian Charter of Rights and Freedoms (section 15), the United Nations Convention on the Rights of Persons with Disabilities (UNCRPD), and provincial human rights legislation requiring reasonable accommodation for persons with disabilities. Canada's 2010 ratification of the UNCRPD marks a fundamental change in the way Canadian society views persons with disabilities in that they are "no longer considered to be recipients of charity or objects of others' decisions, but holders of rights."3 This rightscentric view would apply equally to MCS and VS patients as to any other group with disabilities. Given Canada's moral and legal commitment to persons with disabilities, the evidence of awareness in some patients with disorders of consciousness, and the results of prior phases of research that suggest broad conceptual support from physicians, researchers, and legal experts, ${ }^{4}$ we believe that Canada, as a society, is obliged to explore whether and how neuroimaging technology could be made available to patients with DoC. To this end, we prioritize the following steps for action, that both newly complement and build upon recommendations advanced by other scholars.

\section{RESEARCH}

1. Correlate neuroimaging results with behavioural measures. The Glasgow Coma Scale, the Coma Recovery Scale Revised, and the Wessex Head Injury Matrix are behavioural measures used to assess patients with disordered consciousness. Even with these tools, historically high rates of misdiagnosis underscore the need for empirically validated measures across all substages of the injured brain.

2. Establish incidence and prevalence of MCS and VS. Data on the course of recovery from coma through VS and MCS to normal consciousness are essential to augment sparse current statistics.

3. Determine the probability that covert consciousness will remain undetected. Signals may go undetected if patients produce neural responses that are too subtle or slow to be detected or if patients do not respond for another reason unrelated to awareness. These clinical-technical challenges underscore the importance of mitigating false-negative results.

4. Harmonize methods, paradigms, and selection of stimuli. Harmonized methods and acquisition paradigms will ultimately yield the greatest power for statistical analysis and comparison to neuropsychological evaluation.

5. Assess the possibility of developing quality-of-life metrics. Assessing quality of life of patients with covert consciousness is a difficult challenge given the limited envelope for communication, but any reproducible strategy will serve to inform the state of these patients and assist health care system decision-making.

\section{Translation}

1. Develop knowledge translation on the meaning and implications of covert consciousness. Specialized knowledge translation tools are essential to capturing and communicating the phenomenon of consciousness after severe brain injury and promoting understanding among physicians, allied health professionals, the media, and the general public.

2. Establish guidelines for timing neuroimaging tests. Timing guidelines should consider many factors, including the nature and severity of the injury, behavioural assessments, and the intended use of the test. They should be aligned with published evidence-based guidelines.

From the National Core for Neuroethics, Division of Neurology, Department of Medicine, University of British Columbia, Vancouver, British Columbia, Canada (JI); The Brain and Mind Institute, Western University, London, Ontario, Canada (AMO);

National Core for Neuroethics, University of British Columbia, Vancouver, British Columbia, Canada (ACB).

Received August 26, 2015. Final Revisions Submitted November 6, 2015 Correspondence to: Judy Illes, Faculty of Medicine, University of British Columbia, 2211 Wesbrook Mall, Koerner S124, Vancouver, BC, Canada V6T 2B5.

Email: jilles@mail.ubc.ca 
3. Develop best practices and standards of care for supporting decision-making. Standards for care should be based on quantitative and qualitative research about the reaction of families to choices about testing, their interpretation of the findings, and their strategies to cope with the results. Resulting standards should inform how attending physicians, ethicists, and allied health professionals interact with these families.

4. Develop communication and neuroscientific assessment protocols effective within technical and patient limitations. Study designs involving functional magnetic resonance imaging or other techniques, such as electroencephalography and magnetoencephalography, involve stimulus-response paradigms of varying intervals. Questions must be finetuned to allow patients to successfully fulfill the demands of imaging protocols.

5. Develop protocols appropriate for communicating risks and decisions. Patients must be able to confirm their understanding of the nature and risks of any medical or personal decisions they are asked to make, and their choice must be consistent and temporally reliable.

6. Harmonize terminology used to classify covert consciousness. As new DoC nosologies emerge, new terms and scales for patient management must follow. Harmonization of classification terminology today will mitigate the diagnostic confusion and variability seen in the past.

\section{Canadian Health Care and Legal Systems}

1. Integrate respect for the personhood of patients with DoC. New educational strategies must be developed, tested, and refined to enable the professional community to embrace signals of patients' will and preferences expressed through neuroimaging techniques.

2. Inventory facilities and staff across Canada. Establish a trans-Canadian inventory of facilities and staff needed for assessments of covert consciousness.

3. Clarify the application of capacity laws in the case of DoC. Unique legal questions raised by covertly conscious patients should be addressed to ensure that practice complies with existing legislative and treaty (UNCRPD) obligations. Article 12 of the UNCRPD commits Canada to take "appropriate measures" to enable persons with disabilities to exercise their legal capacity. Although Canada has reserved the right under the UNCRPD to continue to use substitute decision-making regimes where appropriate, it has also indicated that "persons with disabilities are presumed to have legal capacity." Therefore, it is necessary to address the question of what steps are required by the law to overcome the presumption, given the possibility of covert consciousness.

4. Establish criteria for evaluating fairness and distributive justice. A set of common criteria will assist decision-makers to accommodate regional circumstances and budgets while retaining publicly defensible accountability-for-reasonableness frameworks.

Canada has enacted legislation and ratified an international convention that morally and legally obliges society to respect the human rights of persons with disabilities. We have identified priority challenges for research, translation, and policy that must be resolved to enable patients with DoC to fully exercise their rights by providing them with neuroimaging technology to express their will and preferences.

\section{Members of THE MCS NeUroimaging Workgroup}

Judy Illes, National Core for Neuroethics, Division of Neurology, Department of Medicine, University of British Columbia

Adrian M. Owen, The Brain and Mind Institute, Western University

Adrian C. Byram, National Core for Neuroethics, University of British Columbia

Michael Bach, Canadian Association for Community Living

Lara Boyd, Department of Physical Therapy, University of British Columbia

Jennifer Chandler, Faculty of Law, University of Ottawa

Joseph J. Fins, Division of Medical Ethics and Consortium for the Advanced Study of Brain Injury, Weill Cornell Medical College

Joseph T. Giacino, Department of Physical Medicine and Rehabilitation, Harvard Medical School

Teneille Gofton, Department of Clinical Neurological Sciences, Western University

Grace Lee, National Core for Neuroethics, University of British Columbia

David K.B. Li, Department of Radiology, University of British Columbia

Eric Racine, Institut de recherches cliniques de Montréal

Alexander Rauscher, University of British Columbia MRI

Research Centre, Department of Pediatrics

Urs Ribary, Cognitive Neuroscience in Childhood Health and Development, Simon Fraser University

Christine Stables, Division of Neurology, University of British Columbia

A. Jon Stoessl, Division of Neurology, University of British Columbia

Andrea Townson, Division of Physical Medicine and Rehabilitation, University of British Columbia

David G.T. Whitehurst, Faculty of Health Sciences, Simon Fraser University

\section{Other Participants and ACKnowledgments}

Laura Cabrera, National Core for Neuroethics, University of British Columbia

Richard Nakamura, Center for Scientific Review, National Institutes of Health

Susan Zimmerman, Secretariat on Responsible Conduct of Research, CIHR, Natural Sciences and Engineering Research Council, and Social Sciences and Humanities Research Council

\section{ACKNOWLEDGMents AND FUnding}

Supported by Canadian Institutes of Health Research (CIHR): EOG \#120257 and CNE \#85117, and the Canada Chairs Program (JI). AO received research support from Canada Excellence Research Chairs Program, CIHR, Canada Foundation for Innovation grant, National Sciences and Engineering Research Council (NSERC) Discovery grant, James S. McDonnell Foundation grant, and NSERC Research Tools and Instruments grant. UR received research support 
from BC Leading Edge Endowment Fund and Canada Foundation for Innovation (CFI) and Operating (CFI-IOF) grant.

\section{Disclosures}

JI served as principal investigator (grantee) for and received research support from Canadian Institutes of Health Research (CIHR) CNE85117 and EOQ120257. AO served as principal investigator for and received operating grants from CIHR and NSERC. AB has nothing to disclose.

\section{REFERENCES}

1. Owen AM, Coleman MR, Boly M, Davis MH, Laureys S, Pickard JD. Detecting awareness in the vegetative state. Science. 2006; $313: 1402$
2. Monti MM, Vanhaudenhuyse A, Coleman MR, Boly M, Pickard JD, Tshibanda L, et al. Willful modulation of brain activity in disorders of consciousness. N Engl J Med. 2010;362:579-89.

3. Office of the High Commissioner for Human Rights. Monitoring the convention on the rights of persons with disabilities. Guidance for human rights monitors. Professional training series no. 17. New York and Geneva; 2010. http://www.ohchr.org/documents/ Publications/disabilities_training_17en.pdf.

4. Lee G, Byram AC, Owen AM, Ribary U, Stoessl AJ, Townson A, et al. Canadian perspectives on the clinical actionability of neuroimaging in disorders of consciousness. Can J Neurol Sci. 2015;42:96-105.

5. Convention on the Rights of Persons with Disabilities: Declaration and Reservation by Canada. Treaty Ser., vol. 2515, New York, NY: United Nations; 2008. 